\title{
ADDITION TO MR WALTON'S PAPER “ON THE RAY-PLANES IN BIAXAL CRYSTALS.”
}

[From the Quarterly Journal of Pure and Applied Mathematics, vol. XII. (1873), pp. 273-275.]

INSTEAD of $\mathrm{Mr}$ Walton's $a^{2}, b^{2}, c^{2}$ write $a, b, c$, and assume $\alpha, \beta, \gamma=b-c, c-a$, $a-b ; \delta, \epsilon, \zeta=b+c, c+a, a+b$. Also instead of his $x^{2}, y^{2}, z^{2}$ write $x, y, z$. Then instead of the octic cone we have the quartic cone, or say the quartic curve

$$
\begin{aligned}
& \alpha^{2} \beta^{2} \gamma^{2} \sec ^{2} \theta . x y z(x+y+z) \\
= & \alpha^{2} y z\left\{\left(b c-a^{2}\right) x+a(\beta y-\gamma z)\right\}^{2} \\
+ & \beta^{2} z x\left\{\left(c a-b^{2}\right) y+b(\gamma z-\alpha x)\right\}^{2} \\
+ & \gamma^{2} x y\left\{\left(a b-c^{2}\right) z+c(\alpha x-\beta y)\right\}^{2}
\end{aligned}
$$

viz. we may herein consider $x, y, z$ as trilinear coordinates, the ratios $x: y: z$ being positive for a point within the fundamental triangle.

The curve passes through the angles of the triangle, and it touches the sides in the points $(x=0, \beta y-\gamma z=0),(y=0, \quad \gamma z-\alpha x=0), \quad(z=0, \quad \alpha x-\beta y=0)$ respectively. Moreover, the tangents at the angles of the triangle lie each of them outside the triangle. Hence, supposing $a, b, c$ each positive, and $a>b>c$, we have $\alpha$ and $\gamma$ each positive, $\beta$ negative, and the form of the curve is as shown in the figure, or else the like form with the oval lying outside the triangle. And it is hence clear that, if the side $A C(y=0)$ instead of touching the curve meets it in a node, this is a conjugate point arising from the evanescence of the oval; and in this case no part of the curve lies within the triangle. Now considering any point $x: y: z=l: m: n$, we obtain a tetrad of points $x: y: z= \pm \sqrt{ }(l): \pm \sqrt{ }(m): \pm \sqrt{ }(n)$ on the octic cone or curve; and in order that the point on the octic curve may be real, we must have $l, m, n$ all of the 
same sign; that is, the point on the quartic curve must lie within the triangle. Hence, when in the quartic curve the oval becomes a conjugate point, the octic curve has no real branch, but it consists wholly of conjugate points; viz. it consists of the points $A, B, C$ as conjugate points; two imaginary conjugate points answering to the

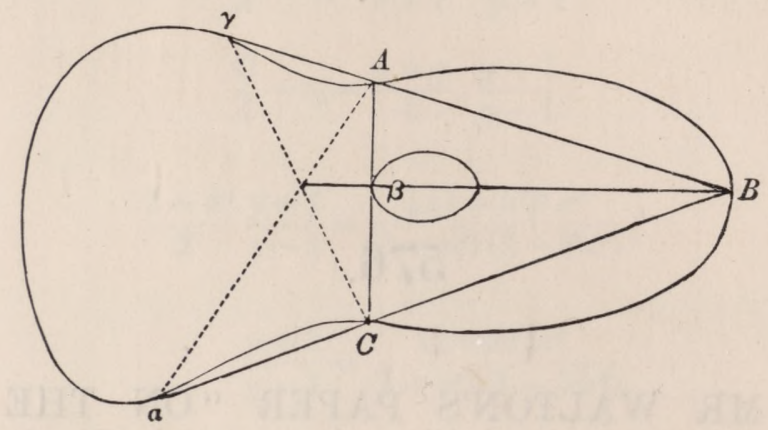

point $\alpha$ of the figure, two other imaginary conjugate points answering to the point $\gamma$; and two conjugate points answering to the point $\beta$, these last being not ordinary conjugate points, but conjugate tacnodal points, or points of contact of two imaginary branches of the curve.

The case in question, $\beta$ a conjugate point on the quartic curve, answers to Mr Walton's critical value of $\sec ^{2} \theta$, viz. in the present notation $\sec ^{2} \theta=\frac{4 b^{2}+\alpha \gamma}{\alpha \gamma}$. To show this I consider the intersection of the curve by the line $\gamma z-\alpha x=0$; and I write for convenience $\gamma z=\alpha x=\gamma \alpha u$, that is, $x=\gamma u, z=\alpha u$. Substituting these values, the equation divides by $y u$, or omitting this factor it is

$$
\begin{aligned}
& \alpha^{3} \gamma^{3} \beta^{2} \sec ^{2} \theta \cdot u\{y+(\alpha+\gamma) u\} \\
= & \alpha^{3}\left\{\gamma u\left(b c-a^{2}-a \alpha\right)+a \beta y\right\}^{2} \\
+ & \beta^{2} \alpha \gamma \cdot u y\left(c a-b^{2}\right)^{2} \\
+ & \gamma^{3}\left\{\alpha u\left(a b-c^{2}+c \gamma\right)-c \beta y\right\}^{2},
\end{aligned}
$$

or observing that we have $\alpha+\gamma=-\beta, b c-a^{2}-a \alpha=\zeta \beta, a b-c^{2}+c \gamma=-\delta \beta$, this becomes

viz. this is

$$
\begin{aligned}
& \alpha^{3} \gamma^{3} \sec ^{2} \theta u(y-\beta u) \\
= & \alpha^{3}(\gamma \zeta u+a y)^{2} \\
+ & \alpha \gamma\left(c a-b^{2}\right)^{2} u y \\
+ & \gamma^{3}(\alpha \delta u+c y)^{2},
\end{aligned}
$$

$$
\begin{aligned}
& u^{2}\left\{\alpha^{3} \gamma^{2} \zeta^{2}+\alpha^{2} \gamma^{3} \delta^{2}+\alpha^{3} \gamma^{3} \sec ^{2} \theta \cdot \beta\right\} \\
+ & u y\left\{2 \alpha^{3} a \gamma \zeta+2 \gamma^{3} c \alpha \delta+\alpha \gamma\left(c a-b^{2}\right)^{2}-\alpha^{3} \gamma^{3} \sec ^{2} \theta\right\} \\
+ & y^{2}\left(a^{2} \alpha^{3}+c^{2} \gamma^{3}\right)=0 .
\end{aligned}
$$


The required condition is that the coefficient of $u^{2}$ shall vanish; viz. we then have

that is,

$$
\begin{aligned}
-\alpha \beta \gamma \sec ^{2} \theta & =\alpha \zeta^{2}+\gamma \delta^{2} \\
& =(b-c)(a+b)^{2}+(a-b)(b+c)^{2} \\
& =(a-c)\left\{3 b^{2}+b(a+c)-a c\right\} \\
& =-\beta\left(4 b^{2}+\alpha \gamma\right),
\end{aligned}
$$

$$
\alpha \gamma \sec ^{2} \theta=4 b^{2}+\alpha \gamma,
$$

agreeing with $\mathrm{Mr}$ Walton's value. Giving $\sec ^{2} \theta$ this value, and throwing out the factor $u$, the equation becomes

$$
\begin{aligned}
& u\left\{2 \alpha^{3} a \gamma \zeta+2 \gamma^{3} c \alpha \delta+\alpha \gamma\left(c a-b^{2}\right)^{2}-\alpha^{2} \gamma^{2}\left(4 b^{2}+\alpha \gamma\right)\right\} \\
+ & y\left(a^{2} \alpha^{3}+c^{2} \gamma^{3}\right)=0
\end{aligned}
$$

or, what is the same thing,

$$
\begin{aligned}
& \alpha \gamma u\left\{2 a(a+b)(b-c)^{2}+2 c(c+b)(b-a)^{2}+\left(c a-b^{2}\right)^{2}-(b-c)(a-b) 4 b^{2}-(b-c)^{2}(b-a)^{2}\right\} \\
& +y\left(a^{2} \alpha^{3}+c^{2} \gamma^{3}\right)=0,
\end{aligned}
$$

say

$$
\alpha \gamma K u+\left(a^{2} \alpha^{3}+c^{2} \gamma^{3}\right) y=0,
$$

viz. this equation determines the remaining intersection of the curve by the line $\gamma z-\alpha x=0$; the point in question lies outside the triangle, that is, $u: y$ should be negative; or $\alpha, \gamma, a^{2} a^{3}+c^{2} \gamma^{3}$ being each positive, we should have $K$ positive; we in fact find

$$
\begin{aligned}
K & =4 b^{4}+b^{2}\left(a^{2}+c^{2}-6 a c\right)+4 a^{2} c^{2} \\
& =4\left(b^{2}-a c\right)^{2}+b^{2}(a+c)^{2}
\end{aligned}
$$

which is as it should be. 